\title{
Surface Electric Field Variations due to Different Human Postures for Wireless Endoscopy Applications
}

\author{
Yana. A. Salchak, Student Member, IEEE, Noor M. Albadri, Matthew T.O. Worsey, Hugo G. \\ Espinosa, Senior Member, IEEE, and David V. Thiel, Senior Member, IEEE
}

\begin{abstract}
RSSI based localization methods for wireless capsule endoscopy (WCE) application requires a patient to wear on-body sensors detecting the RF signals from the capsule. The main advantage of WCE is to allow the patient to live normally and to move freely during the natural transit time, which could take up to 73 hours. Changes in posture can be accompanied by the movement of soft tissues in the abdominal region. This will affect the propagation path length between on-body receiving sensors and the ingested wireless capsule, and consequently the received signal strength (RSS). This paper explores potential variations in RSSI measurements created by postural change. Retro-reflective markers placed in direct contact with the skin of a participant were used to represent appropriate locations of the on-body slot antennas, which could be used for WCE localization. Optical motion capture technology was used to determine an estimate of the location of the markers and corresponding skin position for different postures. The relative displacement of the markers from their initial location (the standing posture) was a maximum of $24 \mathbf{~ m m}$ for the lying posture (supine position) for the participant with BMI of $29 \mathrm{~kg} / \mathrm{m}^{2}$. The maximum variation of the surface electric field of $13.77 \mathrm{~dB}$ was calculated using the attenuation path loss (PL) model for the human abdominal region propagation environment at $2.45 \mathrm{GHz}$. If not anticipated, posture induced variations in RSSI can produce misleading interpretations, and will require adjustments of WCE localization algorithms.
\end{abstract}

Index Terms - wearable sensors, motion capture, soft tissue artefacts , waist girth, propagation path change, RSSI error

\section{INTRODUCTION}

$\mathrm{C}$ onfidence in the received results and accuracy of the measurements are crucial when it comes to medical equipment. They influence decisions taken by medical practitioners, and an incorrect interpretation reduces the effectiveness of the treatment and can even lead to detrimental outcomes.

Manuscript received XXXX, 2021; revised XXX; accepted XXXX, 2021. Date of publication XXX; date of current version September 1, 2021.

Yana .A. Salchak, Noor M. Albadri, Matthew T.O. Worsey, Hugo G. Espinosa, and David V. Thiel are with the School of Engineering and Built Environment, Griffith University, 170 Kessels Rd, Nathan QLD, 4111, Brisbane, Australia (e-mail: iana.salchak@griffithuni.edu.au; noor.albadri@griffithuni.edu.au; matthew.worsey@griffithuni.edu.au, h.espinosa@griffith.edu.au, d.thiel@griffith.edu.au). Digital Object Identifier XXXXXX
Modern technological advances allow the development of medical devices that aim to provide personalized, comfortable and timely diagnostics. To achieve this, medical devices must be portable, not require immobilization of the patient and collect verifiable data during normal daily activities. For instance, wearable sensors are used for monitoring various physiological parameters and further, medical practitioners can interpret the obtained results remotely (electrocardiography (EEG), electroencephalography (ECG), heart rate and oxygen monitoring, endoscopy, etc.). However, when using systems that give patients the freedom of movement, measurements are less controlled and change in body posture and overall motility can lead to the corresponding measurement errors.

Appropriate channel models are of utmost importance for designing reliable and efficient system for medical applications, and the influence of body movement on the measurements is one of the factors that needs to be included. For instance, one of the emerging fields of intra-body communication exploits the human body as a transmission channel, allowing the sensors located on-body or in close proximity to communicate wirelessly. In this case, the signal can be transmitted through the body or through the air depending on the distance between the sensors and the operating frequency. Current research in this field reports that changes in body posture can influence the received signal levels and this effect is more prominent for higher frequencies when propagation is through the body [1] The position of the on-body sensors can change due to the posture of a patient (e.g. lifting an arm), this in turn influences the distance between the communicating nodes and thus needs to be considered when designing an efficient intra-body communication channel [2-3].

For in-body to on-body communication channels, the position of the sensors due to posture change not only affects the performance of the system, but can also produce significant artefacts creating misleading results for applications based on field strength and time-of-flight analysis. In-body communications includes a natural signal source (e.g. the heart) or an implantable transmitter with receiving sensors placed on the skin of the patient (glucose and bladder pressure monitoring, cardiac functions monitoring, wireless endoscopy, etc). For these types of systems, it is assumed that on-body receivers only record the data from the embedded sensor. 
Hence, the position of the sensors should be stable and not affect the obtained measurements.

The most commonly used health monitoring surface sensors are gelled metal electrodes, such as ECG sensors. These sensors are prone to artefacts due to the stretching of the skin [4-6]. Motion artefacts and methods for their reduction are studied in terms of electrocardiographic interpretations and false reading prevention. Variations in the appearance of the $\mathrm{P}, \mathrm{QRS}$, and $\mathrm{T}$ time waveforms are evaluated and special design configurations such as signal adaptive filtering, and more recently advanced signal processing algorithms are used to detect and eliminate false signals. [7-9]. These parameters change with skin properties, such as skin potential reduction. On the other hand, there are cases where the displacement of the sensor itself can lead to measurement errors. For instance, in human movement analysis where skin markers are used for tracking the joint or skeletal motion. The research shows that skin deformations attributed to soft tissues artefacts create discrepancies with the actual bone structures movement [10-12]. For in-body to onbody communication channels, such displacement of the soft tissues means the signal propagation distance changes. This can reduce the corresponding signal path loss estimation reliability.

Wireless capsule endoscopy (WCE) is a recently developed technique for the gastro-intestinal (GI) diagnostics by implantation of a miniaturized capsule (the pill) that propagates through the entire GI tract of the patient while recording the video of the lining. At present, the receiving technology involves ECG- type sensors placed on the skin to collect and record video data transmitted from the capsule [13]. The current technology does not provide localization of the pill, which complicates the interpretation of the video results and reduces opportunities for further targeted treatment. Solutions based on various physical principles are studied in order to develop an efficient localization technique. Radio signal strength indicator (RSSI) localization methods are of great interest as they are based on analysis of the same RF signals that are used for data transmission and do not require any design modifications [14]. Receiving antennas are placed on the skin of the patient in the abdominal region. Different wearable onbody antennas designs are currently being investigated by several research groups. In our research, we use a small slot antenna of $2.45 \mathrm{GHz}$ (ISM Band) (Fig. 1). The antenna design and characteristics were previously published and verified in [15] demonstrating that almost all energy radiates through the

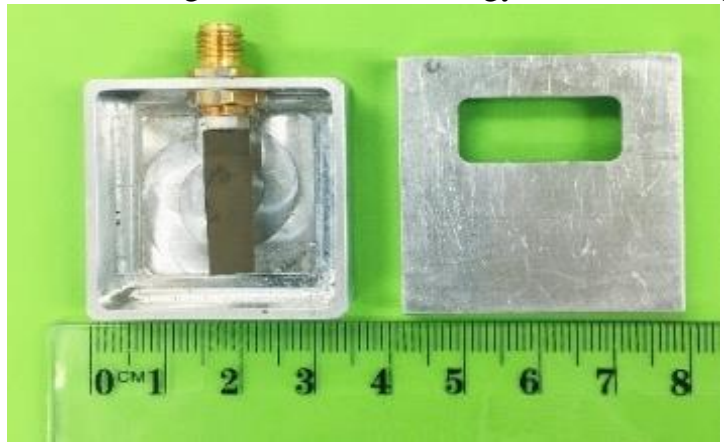

Fig. 1. The slot antenna with the frequency of $2.45 \mathrm{GHz}$. The dimensions of the aluminium box: $11 \mathrm{~mm} \times 33 \mathrm{~mm} \times 33 \mathrm{~mm}$, the dimension of the brass: 5 $\mathrm{mm} \times 21.50 \mathrm{~mm}$.

slot of the antenna inwards with minimal interference from objects holding the back of the antenna (front-to back isolation higher $-15 \mathrm{~dB}$ ). The in-vivo validation of antenna performance demonstrated consistent results when tested on human skin of several participants with various anthropometric parameters [16]. The paper concluded that the field strength measurement error was less than $2 \mathrm{~dB}$ for all positions and all participants.

Received signal strength values can serve to predict the distance to the ingested capsule from the receiving antenna with known coordinates. In order to estimate the spatial (3D) location of the ingested capsule inside the human abdomen region, signals recorded by several receiving antennas are used in conjunction with triangulation and trilateration methods as well as optimization techniques.

It is crucial to have a reliable propagation model that gives an exact correspondence between the signal path loss and the distance from the capsule to each of the measurement points. Within this model, the positions of the receivers are fixed and any change in the RSSI is caused by the capsule passage inside the GI tract. The main contribution to the path loss is the radio attenuation in the soft tissues of the abdominal region of the patient. An unpredicted displacement of the soft tissues could potentially lead to the path length variation that does not reflect the capsule movement, but rather the change of the propagation model parameters and receiving on-body sensors displacement. This can be considered as artefacts or noise, as in that case the obtained RSSI values will no longer correspond to the measurement points that were included in the initial model.

Several studies on WCE localization using magnetic tracking, discuss the importance of the position of the receivers [17-18]. For methods based on magnetic fields analysis, the noise is created by geomagnetism and magnetic disturbance in the environment. In [18] SNR analysis is performed for each sensor measurement position to define the threshold and adjust the priority of measurements to those within the threshold by assigning them special weights. The paper provides guidance for WCE magnetic tracking systems design considering the presence of noise.

This paper presents the first attempt to investigate the surface electric field strength variations caused by postural change that should be accounted for when designing RSSI based localization systems for WCE applications. In order to represent normal daily activity of the patient various body postures are tested by using motion capture technology. The results provide a quantitative analysis of soft tissue displacement in the abdominal region, and the corresponding variation of RSSI values. Evaluation of the skin displacement for each on-body receiver position can serve as an estimate of their individual reliability and further be incorporated within the capsule location retrieving algorithm for the participant of this trial. For general guidelines on the distribution of the receivers for RSSI based localization systems accuracy improvement, a larger group of participants are to be studied in future work.

\section{Abdominal Tissues Displacement Measurements}

Keith [19] first established the displacement of the organs in the abdominal region due to body posture change in pathology research in 1898. Since then, this phenomenon was investigated by various research groups using different approaches 
depending on the applications.

In the engineering field, the research is aimed at development of advanced numerical body models, which include inner organs and their morphology. Improvement of such models is beneficial mainly for ergonomics and injury prevention when designing safe transport vehicles. As commonly used, body models are based on the imaging data gained from patients in the supine position, the available computational human body models do not reflect the deformation and movement of the organs and soft tissues in other postures. Modelling using morphing techniques with corresponding post-mortem surrogate's response to the posture change is one of the common approaches being implemented for these purposes [20-22]. However, due to the primary application of these models, the body posture change to a seated or driving posture has only been considered. The results are commonly represented as the comparison between predicted organ deformations due to loading and the real deformations in the surrogates.

Research involving the comparison of the data gained from medical imaging of the living patients allows creating more accurate models. Positional magnetic resonance imaging (MRI), computed tomography (CT), and ultrasound imaging are being used to obtain images of inner structures for these purposes [23-25]. However, similarly to post-mortem surrogate studies, only two posture positions, seated and the supine were considered as well as only specific organs such as spleen, kidneys and liver deformations. This is due to the organs under the rib cage being most vulnerable during vehicle collision.

Biomedical engineering literature shows the development of abdominal soft tissue models for broader applications, which include comparison of various body postures as well as the movement of the entire abdominal cavity organs.

Lafon et al. [26] performed a broad study using the results of positional MRI to create a model showing deformations of the organs and soft tissues in the trunk region. The displacement of the organs was estimated by using a segmentation modelling approach and validation was performed using the MRI data of one subject. In order to evaluate the displacement of the abdominal cavity (which corresponds to the gut location), the center of gravity position along S1 to T1 spinous processes and along the posterior-anterior axis was estimated, representing horizontal and vertical planes. It was found that the biggest displacements were $4 \mathrm{~mm}$ and $20 \mathrm{~mm}$ correspondingly to each plane due to the posture change. The object shape in that region was defined as the distance from the L4 spinous process to the anterior skin surface, and was estimated to be larger in the seated and the forward-flexed postures than in other postures ( $163 \mathrm{~mm}$ to $183 \mathrm{~mm}$ vs. $100 \mathrm{~mm}$ to $132 \mathrm{~mm}$ ).

Medical research is mainly focused on assessing the displacement of the organs and soft tissues in the abdominal region due to the respiratory movement. This is explained by the most common medical application of these results, such as targeted treatment (e.g. radiotherapy), or assisted surgery. In most cases, the patient is completely or partly immobilized and the body posture does not change significantly during a procedure, although breathing can cause artefacts in corresponding imaging results [27-29].

It is known that the rib cage and diaphragm displacement reflects equal displacement of the abdominal wall and contents [30-31]. This correlation between the respiratory movement of the diaphragm and the organs and soft tissues is used to create advanced numerical models. The displacement of the diaphragm can be measured visually or by using optical tracking methods. For example, mesh surface reconstruction using structured light is used for these purposes by gathering the point cloud data of the whole surface [32-34].

Using preoperative $\mathrm{CT}$ scans and 3D reconstruction by means of structured light, a simulation of the abdominal organs motion during quiet breathing was reported [35]. Positional data of the optical markers along the whole torso surface enabled skin position estimation, and 2D cross-sections in the anteriorposterior direction were used to estimate the displacement of the skin. The important assumption used in this work was that the position of the patient's skin due to breathing is necessary to predict the movement of the organs.

The work presented in our paper focuses on evaluating the reliability of engineering solutions for practical biomedical applications. We suggest evaluating the skin displacement due to the body posture changes as an indicator of the corresponding soft tissues movement in the abdominal cavity. The skin position for several body postures is considered at the points on the torso surface where wearable receiving RF antennas can be placed for wireless endoscopy purposes.

The skin position change can be determined in three dimensions using motion capture technology. Motion capture technologies find wide practical application in biomedical engineering. It is commonly used to create robust systems to track human skeletal motion for athlete performance assessment, neurological disease treatment and monitoring, physiotherapy and assisted targeted medical treatment [37-41]. For our purposes, however it is of interest to use a motion capture system as a tool to gain data for the analysis of the skin position and soft tissue movement, which has not been fully reported previously. Most papers published in this area are focused on the implementation of the results in sports and highperformance wear design and wearable textile health monitoring systems [42-45]. Hence, the analysis was made for the flexing joints of the body, and no assessment of abdominal region soft tissues was included.

More recently, motion capture instruments have been introduced to determine the position of the on -body antennas with higher accuracy for a moving person [46-49]. However, in wireless communications, positioning of the antennas on the body commonly is investigated in terms of its performance for on-body communication channels [50-51]. The displacement of the antennas due to body movement and skin deformation has not been investigated.

We focus on the abdomen region anthropometric parameters change during body movement by analysing motion capture results. 3D positioning measurements for the reference markers attached to the torso skin of a participant provide representation of the receiving slot antennas locations used in WCE localization. A comparison of these positioning data for various 
body postures allowed the calculation of the displacement of each of the antennas position due to the movement of the abdominal soft tissues, and visualize the corresponding waist girth for each postural case.

\section{Methodology}

\section{A. Measurement Setup}

Experiments were performed at the motion capture studio at the School of Engineering and Built Environment, Griffith University, Queensland, Australia. The trials were conducted in accordance with Griffith University's ethics research committee (Ethics approval number GU Ref No: 2018/601). A 69 year old male participant with the BMI of $29 \mathrm{~kg} / \mathrm{m}^{2}$ (see Table 1). This can be considered as overweight, hence more representative in terms of soft tissues movement in the abdominal area.

TABLE I

ANTHROPOMETRIC PARAMETERS OF THE PARTICIPANT

\begin{tabular}{|c|c|c|c|c|c|}
\hline $\begin{array}{l}\text { Height, } \\
\mathrm{cm}\end{array}$ & $\begin{array}{c}\text { Body } \\
\text { mass, kg }\end{array}$ & $\begin{array}{l}\mathrm{BMI}, \\
\mathrm{kg} / \mathrm{m}^{2}\end{array}$ & & irth, cm & \\
\hline \multirow[b]{2}{*}{179} & \multirow{2}{*}{93} & \multirow[b]{2}{*}{29} & Chest & Waist & Hips \\
\hline & & & 100 & 103 & 101 \\
\hline
\end{tabular}

Four different body postures were tested, representing normal movements of patients as part of their daily living activities during the WCE capsule transit time. Assessment of the soft tissues and organ displacement for these postures is required to evaluate any significant changes in the radio propagation path for applications when the receiving on-body antennas are located on the torso, and a transmitting antenna is ingested in the GI tract. This was achieved through comparison of the 3D torso skin position to an initial standing posture. Each of the tested locations on the torso corresponded to a potential on-body slot antenna location and was represented by a retro-reflective marker attached to the skin of the participant.

Three-dimensional positioning data for each of the retro-reflective markers were obtained using an OptiTrack ${ }^{\circledR}$ (www.optitrack.com) motion capture system with passive markers detection using infrared optical sensing. Twelve Flex 3 cameras with a resolution of $640 \times 480,4.5 \mathrm{~mm}$ lenses and field of view of $46.2^{\circ}$ (horizontal) and $34.7^{\circ}$ (vertical) were placed along the perimeter of a $2.6 \mathrm{~m} \times 4.8 \mathrm{~m} \times 2.6 \mathrm{~m}$ (length $\times$ width $\times$ height) capture space. A maximum position error of 0.422 $\mathrm{mm}$ was achieved after camera calibration in accordance with manufacturer's specification. The motion capture system provides continuous $3 \mathrm{D}$ tracking of the retro-reflective markers in the coordinate system of the capture space. The sampling rate of the system was 100 frames per second and the participant was instructed to hold each posture for several seconds. The retro-reflective markers representing the slot antenna locations were placed in the abdominal region on the front and the back of the torso, to obtain measurements along the complete circumference. Two additional markers were used, one on the hand and one on the head of the participant. These markers were not included in the analysis of the skin and soft tissues displacement. The marker on the head served to establish the orientation of the body within the global coordinate system and confirm the posture change for the following data analysis. The hand marker was used to detect when the participants arm position changed within the analysis of a given body posture.

The optical motion capture system setup is represented in Fig. 2, with the participant in the center of the capture space. The red crosses show the location of the additional markers on the head and the hand of the participant. The systems software enabled labeling of the markers accordingly to record individual datasets.

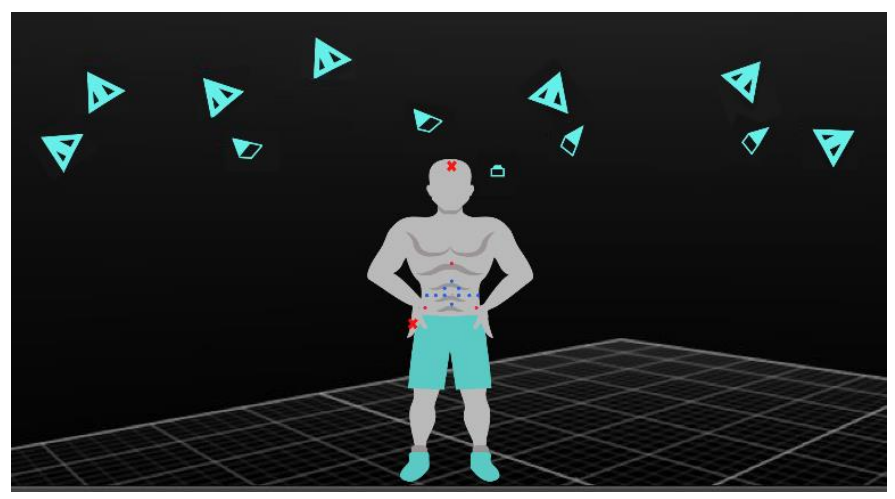

Fig. 2. 3D Representation of the 12-camera setup for the OptiTrack motion capture system used in the study for data acquisition.

A total of 18 retro-reflective markers were placed on the torso of the participant. The locations from 1 to 14 in the abdominal region were distributed along the complete circumference of the torso (Fig. 3, blue). For the anterior sites, the margins of the rib cage were defined first, to avoid bony structures, as well as L3 to L5 spinous processes on the posterior site.

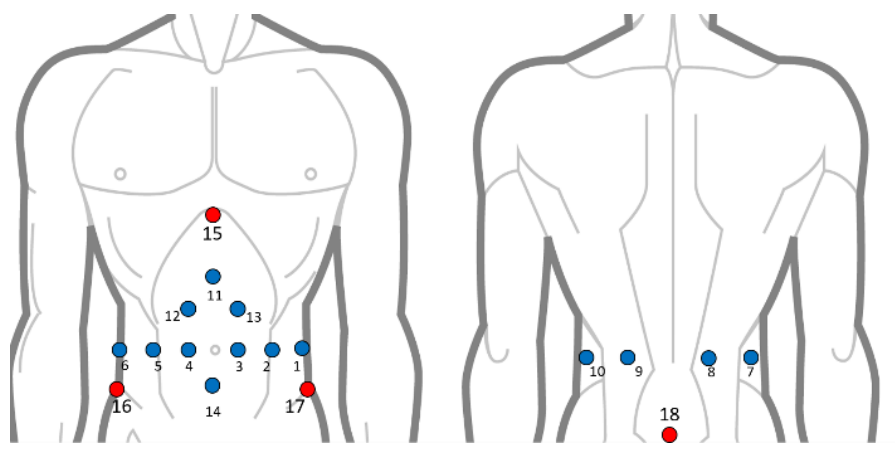

Fig. 3. Representation of the locations of the markers on the skin in the abdominal region. The red dots indicate the locations of reference markers positioned close to bone. The blue markers are positions of interest in GI tract pill tracking and are located on soft tissue regions (slot antenna locations).

Another four reference reflector locations corresponded to the anatomical landmarks near bone (Fig. 3, red), including the sternum (15), pelvis (hip bones 16, 17) and sacrum (18) which show minimal variation during the postural change. The markers numbered from 1 to 10 form the circumference of torso or the waist girth. Their positioning data was acquired continuously, as they were visible to the cameras for every posture, hence they were used for the skin position displacement estimation.

The following four postures were tested:

- Standing posture (Fig. 4a), which was used as the initial position and the displacement of the markers were compared to 
it;

- Seated posture (Fig. 4b),

- Squatting posture (Fig. 4c);

- Supine position (or Lying posture in Fig. 4d), where the participant was lying on a horizontal table on their back and on their front correspondingly, to determine the waist girth skin position measurements of the torso.

Previous research shows that arms position can significantly influence the magnitude of the waist girth [52], therefore this intervention was included for the upright postures (Standing and Seated) as it is demonstrated in Fig. 4(a,b). Within the continuous positioning datasets, the given intervention was identified based on the sudden change of the additional on-hand marker position.

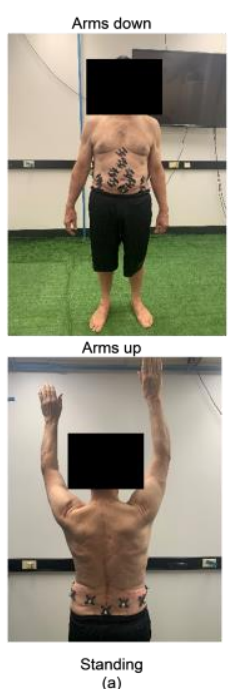

(a)

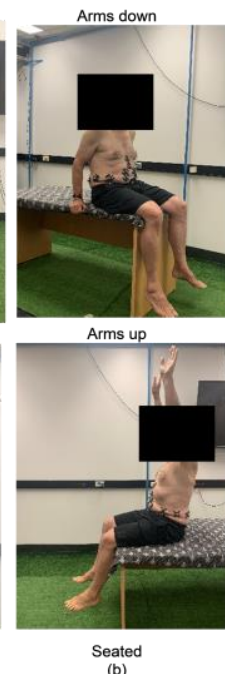

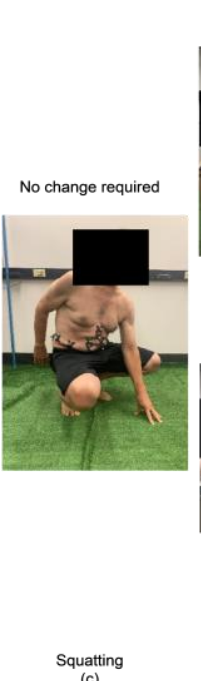

Squattin


Fig. 4. Four body postures with retro-reflective markers on the torso representing daily activity recorded using the OptiTrack Motion Capture System.

\section{B. Antenna Performance and Surface Electric Field Estimation}

The reliability of the cavity backed slot antenna suggested for real case WCE applications was tested in a previous study amongst several participants with various BMIs [16]. It was suggested as an excellent option for the RSSI-based localization methods. The antenna bandwidth is sufficient to accommodate the possible changes amongst the tested locations in the abdominal region, and to minimize the effect on the field strength measurements.

However, each individual has a different constitution and thickness of the skin/fat/muscle layers that could cause a shift in the resonant frequency. Within the current study, we performed reflection coefficient $\left(S_{11}\right)$ measurements to validate the slot antenna performance for the participant at $2.45 \mathrm{GHz}$. The tested antenna locations were congruent with the previous study, although expanded symmetrically to cover the complete waist circumference. This allowed making a more comprehensive judgement on the abdominal region soft tissues movement. The performance of the slot antenna was tested using a vector network analyzer (N9923A Field Fox Handheld
RF VNA $6 \mathrm{GHz}$, Keysight Technologies ®, www.keysight.com) and the acquired $S_{11}$ values are represented in Fig. 5 for both antenna polarizations. The mean $S_{11}$ values were $-7.98 \mathrm{~dB}$ (Standard deviation $1.62 \mathrm{~dB}$ ) for the horizontal orientation of the antenna slot (along transverse anatomical plane); and $-7.01 \mathrm{~dB}$ (standard deviation $1.48 \mathrm{~dB}$ ) for the vertical orientation of the antenna slot (along sagittal anatomical plane). It is evident that for all suggested locations in the abdominal region of the participant the $S_{11}$ values were lower than $-4 \mathrm{~dB}$, which means there is no significant influence on the field strength measurements caused by antenna performance. The change in the propagation path is the main characteristic influencing the RSSI.

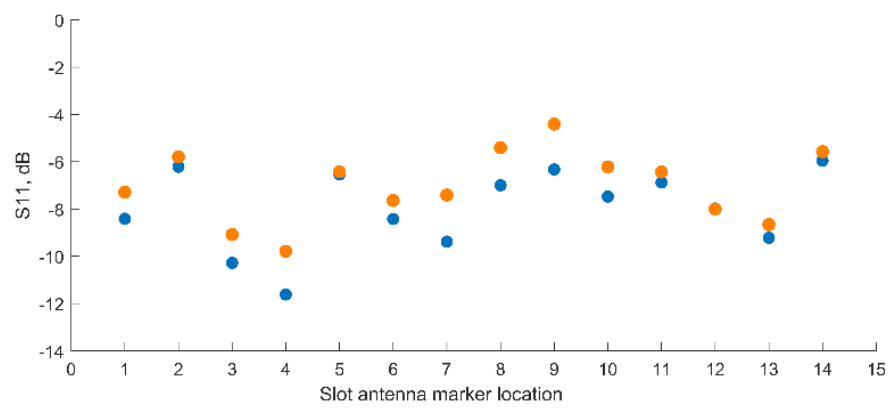

Fig. 5. $\mathrm{S}_{11}$ measurements at $f=2.45 \mathrm{GHz}$ of the slot antenna placed on the torso skin surface at the positions corresponding to the marker locations for the motion capture analysis (blue markers in Fig.3). Note that the orientation of the linearly polarised antenna is included as blue dots (horizontal polarization) and orange dots (vertical polarization).

In general, to predict RSS values, a PL propagation model is required. In a previous work published by the authors [53], the electric field $E$ attenuation PL model was validated for microwave propagation through the lossy medium of the human body:

$$
E \sim \frac{e^{-\alpha R}}{R} \sin \theta_{d}
$$

where $R$ is path length; $\theta_{d}$ is the angle between the dipole source and the surface location and $\alpha$ is the attenuation coefficient. It was validated for the lossy media representing human abdominal region organs with electromagnetic properties at $2.45 \mathrm{GHz}\left(\sigma=2.04 \mathrm{~S} / \mathrm{m} ; \varepsilon_{\mathrm{r}}=53.88, \alpha=51 \mathrm{~Np} / \mathrm{m}\right)$. Since there was no ingested transmitter included in the current study and no real RSSI measurements taken, the PL model was used to evaluate the signal loss with respect to the potential elongation of the propagation path length $R$ due to postural change.

The field strength attenuates logarithmically with distance, and shorter distances between the transmitter and receivers demonstrate higher losses. If transmitter is located closer to the skin surface, the sudden increase in the propagation path will have a larger effect than when the transmitter is located in deep layers of the GI system. In order to make realistic assumptions of the significance of the RSS variation, we considered the initial propagation path length between an ingested capsule and the skin surface prior to the postural change. 
The abdominal wall thickness of the participant was evaluated in order to estimate the minimum possible distance $R_{\min }$ between the transmitter and the receiver on the skin surface. Ultrasound scanning results previously performed on the participant [16], were reassessed in the current study to estimate the thickness of the abdominal wall. The ultrasound imaging system (GE LOGIQe ultrasound unit-General Electric Healthcare ${ }^{\circledR}$, www.gehealthcare.com) was used with a $5 \mathrm{MHz}$ convex array transducer The imaging results show an average skin thickness of $3.00 \mathrm{~mm}$; average fat thickness of $15.50 \mathrm{~mm}$; and the muscle thickness of $20.00 \mathrm{~mm}$ (Fig. 6). Overall, the distance from the skin to the peritoneum is assumed as $38.50 \mathrm{~mm}$. The peritoneum thickness should be no more than $1 \mathrm{~mm}$ in a healthy human, and we assume that the gut wall thickness was $5 \mathrm{~mm}$, as well as the average thickness of the omentum of $15 \mathrm{~mm}$. Therefore, the minimum distance between the surface skin of the torso and the gut (wireless capsule location) $R_{\min }$ is approximately $60 \mathrm{~mm}$ for the participant for the direct propagation path. In terms of the path loss model, this will correspond to the steepest slope or the largest field strength variation for this particular person.



Fig. 6. Transverse ultrasound image of the anterior abdominal wall (each arrow indicates the individual layer of the wall).

Consequently, with an increase of the initial distance, an additional change of the propagation path will cause smaller variations of the received signal strength values. The maximum possible distance $R_{\max }$ between the transmitter and the receiver for the participant was considered. The ultrasound scan is depth limited, hence the $R_{\max }$ that suggested in IV.Results and Discussion Section. $C$ was based on both anthropometric data gathered in the study and basic human anatomy.

The 3-D positioning data for all markers for each body posture were used to estimate skin and soft tissues displacement using numerical calculations in MATLAB ${ }^{\circledR}$ (www.mathworks.com). The maximum total displacement of the markers can be viewed as the maximum change of the propagation path $\Delta R$ and included in the PL calculations.

\section{Data Evaluation and Processing}

All retro-reflective markers were fixed on the skin of the participant, and their position relative to the skin remained stable during postural changes. Hence, we considered the skin position and the markers position as identical, and any deviation from their initial state was influenced by the deformation and displacement of the soft tissues inside the abdominal cavity.

The motion capture system records position data of the markers as a continuous time sequence of their Cartesian coordinate values ( $X, Y, Z$ components), while the participant moves within the capture space. For every posture, including interventions within one posture (Hands Down to Hands Up for the Seated and Standing postures), the position of each of the reflectors in 3D space was determined.

Since the interest of this study was in the positioning information of the skin, and not in the movement of the person inside the capture space, the data for all of the postures were aligned in accordance to a reference point defined by the rigid structures of the participant's body. The position of the centroid point estimated between anatomical landmarks on the torso was used as this reference point having the same coordinates independently of the posture. It was calculated for the initial "Standing Hands Down" posture first, and all other postures datasets were projected onto the same coordinate system using mathematical transformations. This allowed a quantitative assessment of the soft tissue deformations by calculating total displacement of each of the markers in relation to the stable reference centroid point.

The following equation was used to calculate the centroid point coordinates:

$$
\bar{a}_{0}=\frac{1}{n} \sum_{i=1}^{n} a_{0, i}
$$

where $a_{0, i}$ is the vector containing coordinate values $\left(a_{0}=\right.$ $\left.\left[x_{0}, y_{0}, z_{0}\right]\right)$ of the reference markers corresponding to the anatomical landmarks for the initial body posture; $n$ is the number of the reference points used for finding the centroid between them. The sternum and pelvis reference markers (markers 15 to 17 Fig. 3) were used as anatomical landmarks representation, as these had continuous data across all measurements for all postures $(n=3)$.

Several computational steps were undertaken to define the rotation and translation parameters for each posture (or interventions within one):

1) Determine the centroid coordinate values $\bar{b}_{m}$ for a new set of data:

$$
\bar{b}_{m}=\frac{1}{n} \sum_{i=1}^{n} b_{m, n},
$$

where $b_{m}$ is the vector containing coordinate values $\left(b_{m}=\left[x_{m}, y_{m}, z_{m},\right]\right)$ of the reference markers corresponding to any other changed body posture; $m$ in this case represents the number of the datasets for each of the changed body postures ( $m=6$ : Standing. Hands Up; Seated Hands Down; Seated Hands Up; Squatting; Lying Face Up; Lying Face Down); $n$ is the number of reference markers used in the calculation of the centroid point, the same as for the initial position.

The position of the new centroid for each body posture was aligned to the reference centroid $\bar{a}_{0}$ and the coordinates of all the markers were transformed and projected onto the one global coordinate system.

2) The translation vector can be found by subtracting the 
initial posture centroid position from the centroid position of each of the changed body posture:

$$
t_{m}=\bar{b}_{m}-\bar{a}_{0} .
$$

This was further used to modify the complete datasets representing coordinate values of the slot antenna positions markers for each of the changed body postures:

$\left[x_{-}\right.$sensors $_{m}^{\prime}, y_{-}$sensors ${ }_{m}^{\prime}, z_{-}$sensor $\left.s_{m}^{\prime}\right]=$

$\left[x_{-}\right.$sensors $_{m}, y_{-}$Sensors $_{m}, z_{-}$sensors $\left._{m}\right]-t_{m}$,

where $x \_s e n s o r s, y \_s e n s o r s, z \_s e n s o r s$ are datasets of the coordinate values of the markers for a changed body posture (given by $m$ ) before and after transformations correspondingly.

3) For the body postures, where the simple translation was not sufficient due to the anatomical planes of the subject being no longer collinear with the global coordinate system axes, rotation of the local coordinate system was used. A rotation matrix $R T$ in this case was calculated by using Singular Value Decomposition [54]:

$$
\begin{aligned}
& H=\left(b_{m}-\bar{b}_{m}\right)\left(a_{0}-\bar{a}_{0}\right)^{T} \\
& {[U, S, V]=S V D(H)} \\
& R T=V U^{T},
\end{aligned}
$$

where $H$ is the input values matrix, $U$ and $V$ are right and left singular vectors, and $S$ is singular values.

After the reference markers and the centroid positions were aligned, the new locations for all the slot antenna markers along the circumference of the torso were calculated to find the displacement $\Delta R$. The total displacement was calculated using transformed 3D coordinates of each sensor in comparison to its initial state. To visualize the contour along the waist circumference, a smooth curve was fitted between these markers. Each circumference line had 10 points (markers from 1 to 10, Fig. 3), and the interpolation was made by using the built-in spline function in MATLAB ${ }^{\circledR}$.

\section{RESUlTS AND DisCUSSION}

\section{A. Influence of the Arms Position for Standing and Seated Postures}

The coordinate values were processed and the influence of the arms position on the distance between each of the markers to the centroid point was determined. This enabled the displacement estimation. The comparison was made for the Standing and Seated postures between Hand Up and Hands Down.

Fig. 7(a) represents the position of the markers in 3D before and after the participant had raised their hands and the interpolated lines of the circumference at that moment. Fig. 7(b) demonstrates that the arms position change for the Standing posture did not have a significant effect on the overall displacement of the markers along the waist girth. ( $p$-values: 0.928 for $x ; 0.996$ for $y ; 0.188$ for $z$ ).

The same comparison analysis was performed for the Seated posture (Fig. 8). The arms position change for the Seated posture did not have a significant effect on the overall displacement of the markers along the waist girth. ( $p$-values: 0.968 for $x ; 0.972$ for $y ; 0.652$ for $z$ ).

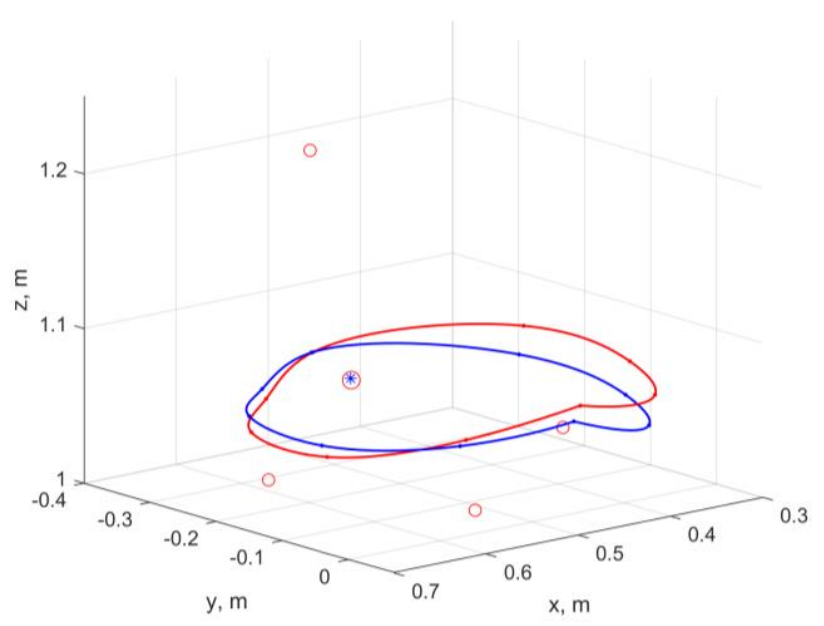

(a)

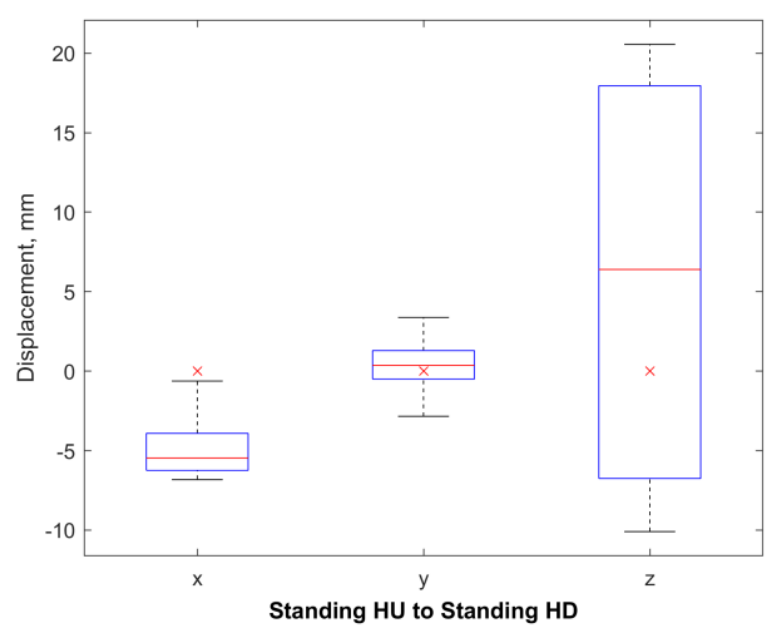

(b)

Fig. 7. (a) Interpolated waist girth lines for the Standing Posture showing the antenna position markers and the reference markers. Hands Down - Red; Hands $\mathrm{Up}$ - Blue; 0 - reference markers for Standing Posture Hands Down, and the centroid position; * - centroid position for Standing Posture Hands Up aligned with the global coordinate system. (b) Mean displacement for all sensor positions (mm) when the participant changed arms position: Standing Hands Down to Standing Hands Up. Direction of the $x$-axis is along anterior-posterior plane, $\mathrm{y}$-axis is along medial-lateral and $\mathrm{z}$-axis is along superior-inferior planes. 


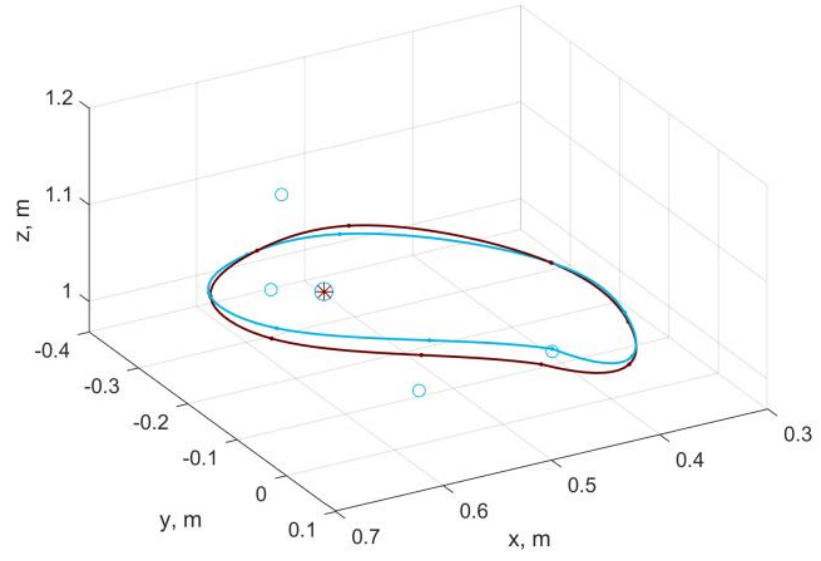

a)

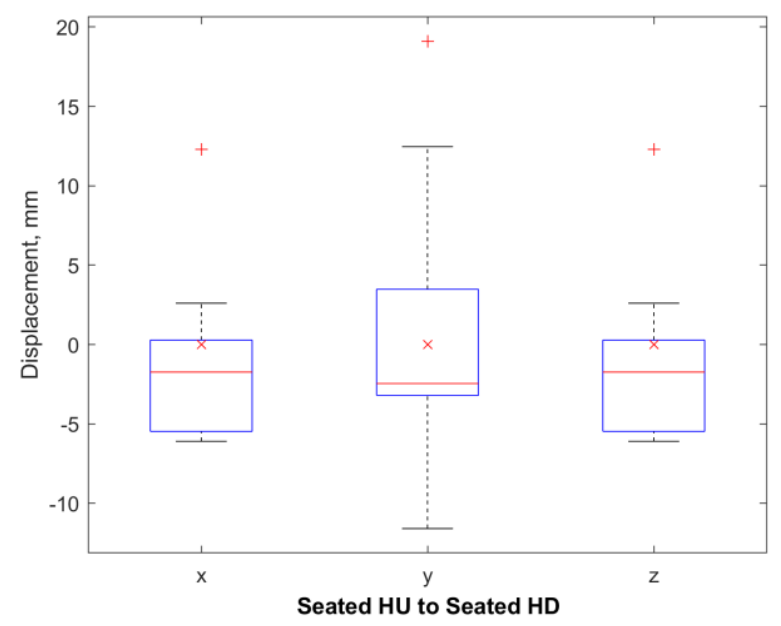

b)

Fig. 8. (a) Interpolated waist girth lines for Seated Posture, Hands Down and Hands Up, showing the antenna position markers and reference markers. Hands Down - Blue; Hands Up -Brown; o - reference markers for Seated Posture Hands Down, and the centroid position aligned with the global coordinate system; * - centroid position for Seated Posture Hands Up aligned with the global coordinate system. (b) Mean displacement along all the sensors ( $\mathrm{mm}$ ) when the participant changed the position from Seated Hands Down to Seated Hands Up. Direction of the $x$-axis is along anterior-posterior plane, y-axis is along medial-lateral and $\mathrm{z}$-axis is along superior-inferior planes.

\section{B. Total Displacement for Each Posture}

The skin position change for each posture was evaluated using positioning data for the retro-reflective markers along the waist girth of the participant. The comparison of the corresponding circumference lines in 3D for all the postures is demonstrated in Fig. 9 and Fig. 10.

The quantitative positional difference was determined to demonstrate the skin displacement and corresponding organs and soft tissues movement. It was calculated as the magnitude of the difference between the distances to the reference centroid point for each slot antenna markers in comparison to such distance for the initial posture. It is shown in Fig. 9 for all 10 markers (which formed the circumference lines), and each given body posture.

The largest skin position displacement from the Standing posture occurred for the Seated and Lying (the supine position)

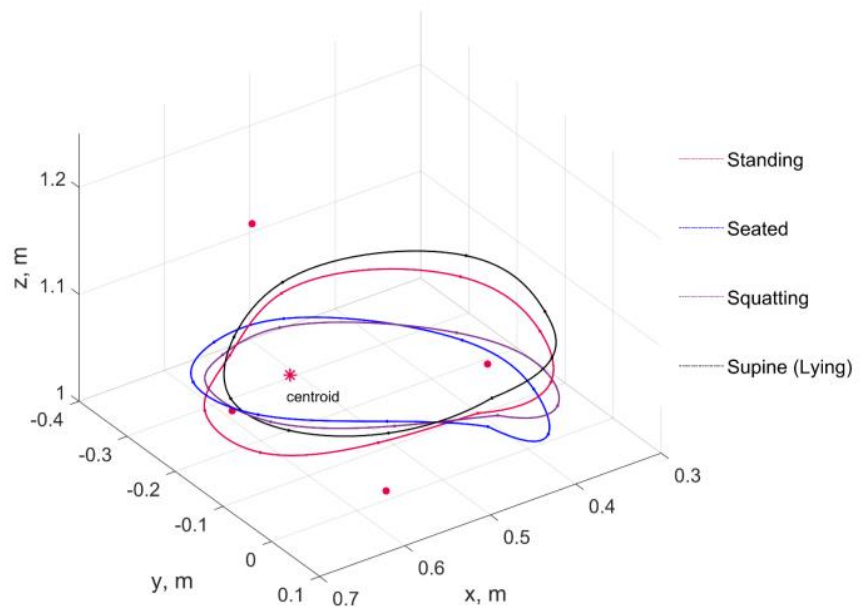

Fig.9 Representation of the circumference torso lines for all four postures in 3D; $*$ - centroid position, $\bullet-$ anatomical landmarks positions.

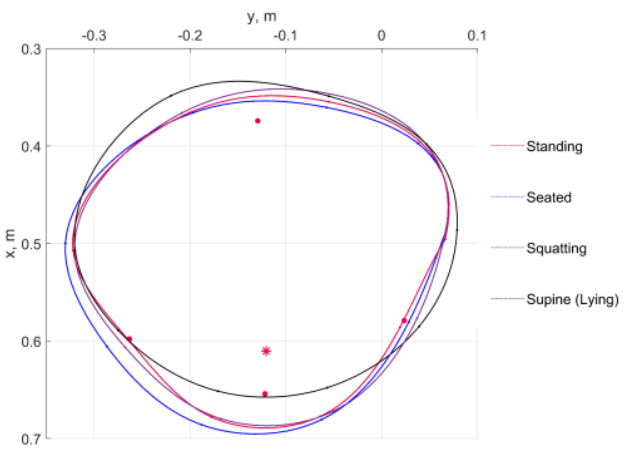

(a)

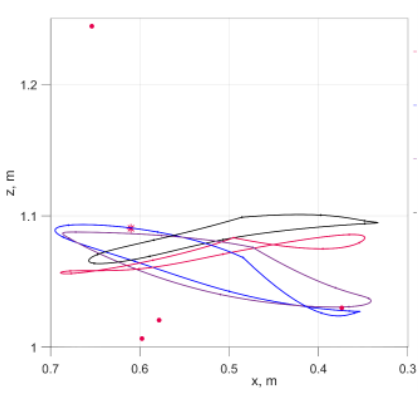

(b)



(c)
Fig.10 Displacement of the interpolated circumference lines (m) compared to the Standing posture Hands Down: (a) $X-Y$ plane; (b) $X$-Z $Z$ plane; (c) $Y$ - $Z$ plane.

postures, which is also evident in the $3 \mathrm{D}$ representation graphs (Fig. 9 and Fig. 10). Fig. 11 demonstrates the largest displacement of the locations 4 and 7 for the Lying posture, (+24 $\mathrm{mm}$ and $-23 \mathrm{~mm}$ respectively). This is congruent to the results reported in [11], with the largest displacement of the abdominal cavity in the horizontal plane. The participant in their studies had smaller BMI of $24 \mathrm{~kg} / \mathrm{m}^{2}$, which can explain slightly smaller skin displacement.

The mean displacement values for each coordinate component across all 10 locations was estimated and is shown in Fig 12 for each posture of the participant compared to Standing, Hands Down. 




Fig. 11. Total displacement of the retro-reflective markers along the waist girth for each antenna location.
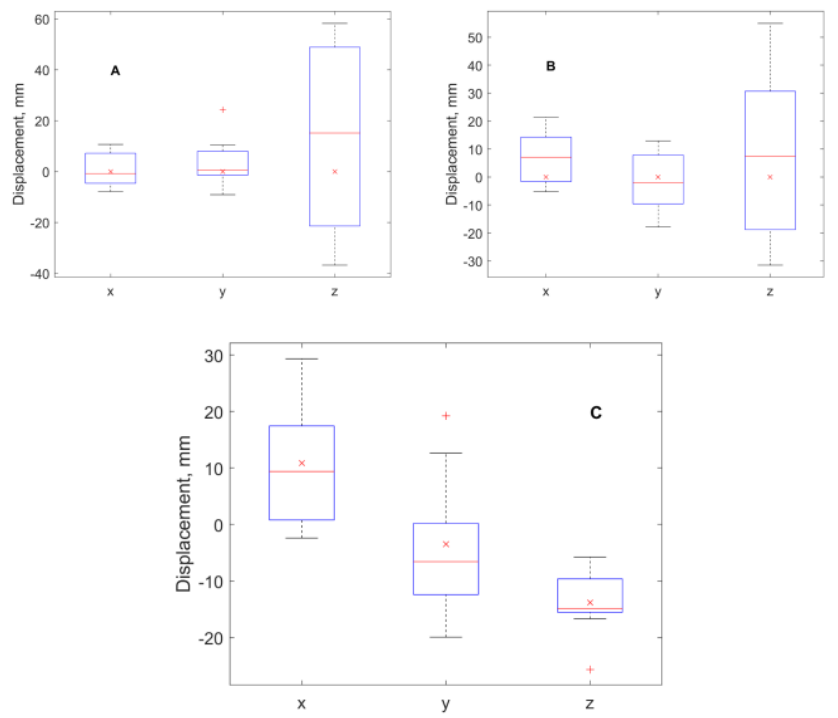

Fig. 12. The mean displacement $(\mathrm{mm})$ for all the 10 sensors locations along circumference for each of the 4 tested postures: (a) Seated; (b) Squatting; (c) Supine (Lying posture).

\section{Analysis of the Surface Electric Field Variations}

The PL model given in Section III.B was used to estimate the field strength values variations in decibels for the predicted maximum soft tissues displacement $\Delta R$ of $24 \mathrm{~mm}$ (Fig. 9).

In that case, the estimated minimum initial distance between the possible location of the ingested transmitter and the on-body sensors will change from $60 \mathrm{~mm}$ to $84 \mathrm{~mm}\left(R^{\prime}{ }_{\text {min }}=R_{\text {min }}+\right.$ $\Delta R$ ). In order to assess the signal variation caused by posture change when the ingested capsule is located at further distances from the on-body receivers, we assumed the $200 \mathrm{~mm}$ distance as the maximum direct propagation length judging by the crosssections of the torso acquired within the study. It is approximately $60 \%$ of the anterior cross section given in Fig. $8 \mathrm{a}$ along the $x$-axis, which corresponds to the anatomical location of the GI tract. Therefore, the variation for the maximum distance between the skin surface and an ingested transmitter is from $200 \mathrm{~mm}$ to $224 \mathrm{~mm}\left(R_{\max }^{\prime}=R_{\max }+\Delta R\right)$.

The relation between the path loss in decibels and the propogation distance in millimeters is given in Fig. 13. It is estimated that the maximum change in the received signal due to the movement of the soft tissues and organs in the abdominal cavity is $13.77 \mathrm{~dB}$, and for the maximum initial distance between the transmitter and the on-body receiver, the value is $11.60 \mathrm{~dB}$

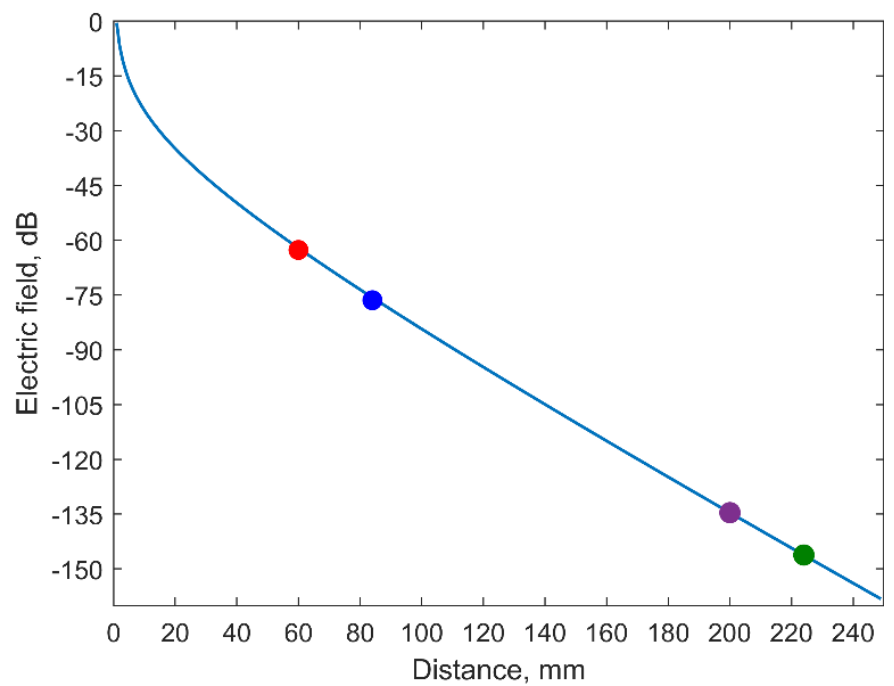

Fig. 13. The relative surface electric field $E$ as a function of the distance or propagation path length to the skin surface from the ingested transmitter (the slope of $-0.69 \mathrm{~dB} / \mathrm{m}$ ): - minimum distance of $60 \mathrm{~mm}, E=-62.66 \mathrm{~dB}$; - minimum distance plus maximum soft tissues in abdominal cavity displacement $-84 \mathrm{~mm}, E=-76.43 \mathrm{~dB} ;-$ maximum distance of $200 \mathrm{~mm}, E$ $=-134.60 \mathrm{~dB} ;-$ maximum distance plus maximum soft tissues in abdominal cavity displacement of $224 \mathrm{~mm}, E=-146.20 \mathrm{~dB}$

\section{CONCLUSION}

Optical motion capture was used to quantify the change in a patient's torso skin position relative to a calculated centroid position for several postures likely to occur during normal living activities. The retro-reflective markers represented the slot antenna locations on the skin surface and their mean displacement compared to the initial standing posture was: Seated: $x=1.07 \mathrm{~mm}, y=3.10 \mathrm{~mm}, z=12.96 \mathrm{~mm}$; Squatting: $x=7.19 \mathrm{~mm}, y=-1.01 \mathrm{~mm}, z=9.68 \mathrm{~mm}$; Lying: $x=10.85 \mathrm{~mm}$, $y=-3.50 \mathrm{~mm}, z=-13.79 \mathrm{~mm}$. The change of the arm positions for standing and seated postures did not cause a significant displacement of the on-body markers.

As the total displacement of the markers (caused by soft tissues and organ movement) was as large as $24 \mathrm{~mm}$, an estimate of the corresponding surface field strength change was as large as $13.77 \mathrm{~dB}$. If such sudden variations caused by postural changes are not accounted for in the post-processing of RSSI, it could lead to poor predictions of the WCE location in real case applications when a wearable receiving system monitoring GI radio signals are worn over a 10-73 hour period [55]. For precise radio pill location based on the received signal strength, either the patient must assume a constant posture, or the change must also be monitored. This information can be further incorporated at the post-processing capsule tracking step. Implementing solutions using a wearable inertial sensors can be considered for monitoring purposes [56].

The error of $24 \mathrm{~mm}$ acquired during the study, is likely to be exacerbated should the measurements be conducted on persons with much larger BMIs. A broader population group should be 
recruited for further statistical analysis.

\section{ACKNOWLEDGMENT}

The authors thank the participant for taking the part in the experiments, and Professor Julie Hides (School of Allied Health Sciences, Griffith University) for her assistance with abdominal ultrasound examination and results interpretation. We also thank the reviewers whose comments and valuable feedback helped to improve and clarify this manuscript.

\section{REFERENCES}

[1] Ž. Lučev, I. Krois, M. Cifrek, M, "Effect of body positions and movements in a capacitive intrabody communication channel from 100 $\mathrm{kHz}$ to $100 \mathrm{MHz}$, Proc. IEEE Int. Instrum. Meas. Technol. Conf., pp. 2791-2795, 2012-May.

[2] T. Kang et al., "Evaluation of human body characteristics for electric signal transmission based on measured body impulse response", IEEE Trans. Instrum. Meas., vol. 69, no. 9, pp. 6399-6411, Sep. 2020.

[3] J. Hwang, T. Kang, Y. Kim and S. Park, "Measurement of transmission properties of HBC channel and its impulse response model", IEEE Trans. Instrum. Meas., vol. 65, no. 1, pp. 177-188, Jan 2016.

[4] L. H. Sigler, "Electrocardiographic changes occurring with alterations of posture from recumbent to standing positions", American Heart Journal, vol. 15 , no. 2, pp. 146-157, 1938.

[5] H. Talhouet and J. G. Webster, "The origin of skin-stretch-caused motion artifacts under electrodes", Physiol. Meas., vol. 17, pp. 81-93, 1996.

[6] A. Y. M. Jones et al., "Changes in heart rate and R-wave amplitude with posture", Chin. J. Physiol., vol. 46, no. 2, pp. 63-70, 2003.

[7] H. W. Tam and J. G. Webster, "Minimizing electrode motion artifact by skin abrasion", IEEE Trans. Biomed. Eng., vol. BME-24, pp. 134-139, 1977.

[8] M. Raya and L. Sison, "Adaptive noise cancelling of motion artifact in stress ECG signals using accelerometer", Proc. Second Joint 24th EMBS/BMES Annu. Conf., vol. 2, pp. 1756-1757, 2002-Oct.

[9] A. Kalra and A. Lowe, "Development and validation of motion artefact rejection system (MARS) for electrocardiography using novel skinstretch estimation approach", Sensors and Actuators A: Physical, vol. 301, p. 111726, 2020.

[10] A. Leardini, L. Chiari, U. Della Croce and A. Cappozzo, "Human movement analysis using stereophotogrammetry: Part 3. Soft tissue artifact assessment and compensation", Gait Posture, vol. 21, no. 2, pp. 212-225, 2005.

[11] N. R. Heneghan and G. M. Balanos, "Soft tissue artefact in the thoracic spine during axial rotation and arm elevation using ultrasound imaging: a descriptive study", Manual therapy, vol. 15, no. 6, pp. 599-602, 2010.

[12] S. Upadhyaya, W.-S. Lee and C. Joslin, "Patient specific bone tracking using ultrasound for human movement analysis", Proc. IEEE Int. MeMeA, pp. 1-5, 2016-May.

[13] D. G. Adler and C. J. Gostout, "Wireless capsule endoscopy", Hosp. Physician, pp. 14-22, May 2003.

[14] D. Hiyoshi and M. Takahashi, "Localization method using received signal strength for wireless power transmission of the capsule endoscope", IEICE Trans. on Commun., vol. 102, no. 8, pp.1660 - 1667, 2019.

[15] M. Fernandez, D. V.Thiel, A. Arrinda, and H.G. Espinosa, "An inward directed antenna for gastro-intestinal radio pill tracking at $2.45 \mathrm{GHz}$ ", Microwave and Optical Technology Letters, vol. 60, no. 7, pp. 1644-1649, 2018.

[16] N. Albadri, J. Hides, H. G. Espinosa, D. V. Thiel, "The effect of human tissue on field strength measurements in vivo using a resonant UHF cavity-backed slot antenna", Bioelectromagnetics, vol. 42 (4), pp. 284295, 2021.

[17] S. Su, H. Dai, S. Cheng, P. Lin, C. Hu and B. Lv, "A robust magnetic tracking approach based on graph optimization", IEEE Trans. Instrum. Meas., vol. 69, no. 10, pp. 7933-7940, Oct. 2020.

[18] G. Shao and Y.-X. Guo, "An optimal design for passive magnetic localization system based on SNR evaluation", IEEE Trans. Instrum. Meas., vol. 69, no. 7, pp. 4324-4333, Jul. 2020.
[19] A. Keith, "A preliminary investigation of the influence of body-posture on the position and shape of abdominal and thoracic organs", Journal of Anatomy and Physiology, vol. 32, no. 3, p. 451, 1898.

[20] P. Beillas, F. Berthet, "An investigation of human body model morphing for the assessment of abdomen responses to impact against a population of test subjects", Traffic injury prevention, vol. 18, no. 1, pp.142 - 147, 2017.

[21] S. Liu, P. Beillas, L. Ding, and X. Wang, "Morphing an existing open source human body model into a personalized model for seating discomfort investigation", presented at the WCX SAE World Congress Experience, SAE Technical Paper no. 2020-01-0874, 2020.

[22] M. K. Howes, W. N. Hardy, A. M. Agnew, and J. J. Hallman, "Evaluation of the kinematic responses and potential injury mechanisms of the jejunum during seatbelt loading", presented at the 59th Stapp Car Crash Conference, SAE Technical Paper no. 2015-22-0009.

[23] A. R. Hayes, F. S. Gayzik, D. P. Moreno, R. S. Martin, and J. D. Stitzel, "Abdominal organ location, morphology, and rib coverage for the 5th, 50th, and 95th percentile males and females in the supine and seated posture using multi-modality imaging", Annals of advances in automotive medicine, vol. 57, p. 111, 2013.

[24] A. R. Hayes, F. S. Gayzik, D. P. Moreno, R. S. Martin, and J. D. Stitzel, "Comparison of organ location, morphology, and rib coverage of a midsized male in the supine and seated positions", Computational and mathematical methods in medicine, vol. 2013, article no. 419821, 2013.

[25] D. Yamada, A. Değirmenci, and R. D. Howe, "Ultrasound imaging characterization of soft tissue dynamics of the seated human body", Journal of Biomechanical Engineering, vol. 142, no. 6. 2020.

[26] Y. Lafon, F. W. Smith, and P. Beillas, "Combination of a modeldeformation method and a positional MRI to quantify the effects of posture on the anatomical structures of the trunk", Journal of Biomechanics, vol. 43, no.7, pp. 1269 - 1278, 2010.

[27] L. Boldrini, D. Cusumano, F. Cellini, L. Azario, G. C. Mattiucci, G. C., and V. Valentini,"Online adaptive magnetic resonance guided radiotherapy for pancreatic cancer: state of the art, pearls and pitfalls", Radiation Oncology, vol. 14, no. 1, pp. 1-6, 2019.

[28] R. Khot, M. McGettigan, J. T. Patrie, and S. Feuerlein, "Quantification of gas exchange-related upward motion of the liver during prolonged breathholding - potential reduction of motion artifacts in abdominal MRI", The British Journal of Radiology, vol. 92, no. 1106, 2020.

[29] M. Pfeiffer, C. Riediger, J. Weitz, and S. Speidel, "Learning soft tissue behavior of organs for surgical navigation with convolutional neural networks", International Journal of Computer Assisted Radiology and Surgery, vol. 14, no. 7, pp. $1147-1155,2019$.

[30] T. I. M. Higenbottam, D Allen, L. Loh, and T. J. H. Clark, “Abdominal wall movement in normals and patients with hemidiaphragmatic and bilateral diaphragmatic palsy", Thorax, vol. 32, no.5, pp. 589-595, 1977.

[31] J. E. R. E. Mead, and S. H. Loring, "Analysis of volume displacement and length changes of the diaphragm during breathing", Journal of Applied Physiology, vol. 53, no. 3, pp. 750 - 755, 1982.

[32] J. Schaerer, A. Fassi, M. Riboldi, P. Cerveri, G. Baroni, and D. Sarrut, "Multi-dimensional respiratory motion tracking from markerless optical surface imaging based on deformable mesh registration", Physics in Medicine \& Biology, vol. 57, no. 2, p.357, 2011.

[33] J. Wasza, P. Fischer, H. Leutheuser, T. Oefner, C. Bert, A. Maier, and J. Hornegger, "Real-time respiratory motion analysis using 4-D shape priors", IEEE Trans. on Biomed. Eng., vol. 63, no. 3, pp. 485 - 495, 2015.

[34] D. Spinczyk, A. Karwan, and M. Copik, "Methods for abdominal respiratory motion tracking", Computer Aided Surgery, vol. 19, no. 1-3, pp. $34-47,2014$.

[35] A. Hostettler, S. A. Nicolau, Y. Rémond, J. Marescaux, and L. Soler, "A real-time predictive simulation of abdominal viscera positions during quiet free breathing", Progress in Biophysics and Molecular Biology, vol. 103, no. $2-3$, pp. $169-184,2010$.

[36] R. S. McGinnis and N. C. Perkins, "A highly miniaturized, wireless inertial measurement unit for characterizing the dynamics of pitched baseballs and softballs", Sensors, vol. 12, no. 9, pp. 11933 - 11945, 2012.

[37] D. Whiteside, B. Elliott, B. Lay, and M. Reid, "The effect of age on discrete kinematics of the elite female tennis serve", Journal of Applied Biomechanics, vol. 29, no. 5, pp. 573 - 582, 2013.

[38] O. Ťupa, A. Procházka, O. Vyšata, M. Schätz, J. Mareš, M. Vališ, and V. Mařík, "Motion tracking and gait feature estimation for recognising Parkinson's disease using MS Kinect", Biomedical Engineering Online, vol. 14, no. 1, p. 97, 2015.

[39] B. K. Weeks, C. P. Carty, and S. A. Horan, "Kinematic predictors of single-leg squat performance: a comparison of experienced 
physiotherapists and student physiotherapists", BMC Musculoskeletal Disorders, vol. 13, no.1, pp. $1-7,2012$.

[40] Radiographic medical imaging system using robot mounted source and sensor for dynamic image capture and tomography, by S. A. Banks, (2008, October 28), Patent US7441953B2.

[41] L. Yahia-Cherif, B. Gilles, T. Molet, and N. Magnenat-Thalmann, "Motion capture and visualization of the hip joint with dynamic MRI and optical systems", Computer Animation and Virtual Worlds, vol. 15, no. 3 - 4, pp. $377-385,2004$.

[42] Z. Hu, L. Liu, Z. Liu, Z., and M. Xing, "Dynamic understanding of human-skin movement and garment design of golf apparel", in International Conference on Applied Human Factors and Ergonomics, pp. 982 - 994, July 2017.

[43] J. Choi, and K. Hong, "3D skin length deformation of lower body during knee joint flexion for the practical application of functional sportswear", Applied Ergonomics, vol. 48, pp. 186 - 201, 2015.

[44] Y. Wang, H. Li, and J. Li, "The skin deformation during cycling on terrains for practical application of compression sportswear", The Journal of The Textile Institute, pp. 1 - 9, 2020.

[45] Y. Wang, and J. Wang, "Design data for running tight: skin strain distribution on lower extremity based on decomposition of movement", The Journal of The Textile Institute, vol. 106, no. 5, pp. 469 - 479, 2015.

[46] A. Brizzi, A. Pellegrini, L. Zhang, and Y. Hao, "Statistical path-loss model for on-body communications at $94 \mathrm{GHz}$ ", IEEE Trans. on Ant. and Propag., vol. 61, no. 11, pp. $5744-5753,2013$.

[47] T. C. Schenk, N. S. Mazloum, L. Tan, and P. Rutten, "Experimental characterization of the body-coupled communications channel", in IEEE ISWCS, pp. 234-239. October 2008.

[48] S. Swaisaenyakorn, S. W. Kelly, and J. C. Batchelor, "A study of factors affecting wrist channel characteristics for walking postures using motion capture", IEEE Trans. on Ant. and Propag.,vol. 62, no. 4, pp. 2231 -2237, 2014.

[49] T. Aoyagi, M. Kim, J.I. Takada, K. Hamaguchi, and R. Kohno, "Body motion and channel response of dynamic body area channel", presented at the 5th EUCAP, IEEE Proc., pp. 3138 - 3142, April 2011.

[50] E. Forrister, G. Lee, D. Xue, B Garner, and Y. Li, "Characterization of narrowband on-body wireless channels using motion capture experimentation", presented at WMCS, IEEE Proc., pp. 1 - 4, March 2016.

[51] Y. Nechayev, C. Constantinou, S. Swaisaenyakorn, O. Rakibet, J. Batchelor, P. Hall and J. Hunt, "Use of motion capture for path gain modelling of millimetre-wave on-body communication links", presented at ISAP, IEEE Proc., pp. 987 - 990, February 2012.

[52] S. C. Lennie, T. Amofa-Diatuo, A. Nevill, and A.D. Stewart, A. D, "Protocol variations in arm position influence the magnitude of waist girth", Journal of Sports Sciences, vol. 31, no.12, pp. 1353 - 1358, 2013.

[53] Y. A. Salchak, H. G. Espinosa, and D. V. Thiel, "Modeling the surface field from an ingested radio transmitter with an approximate attenuation model for gastroenterology investigations", IEEE Trans. on Biomed. Eng., vol. 67, no. 2, pp. $504-511,2019$

[54] K. S. Arun, T. S. Huang, S. D. Blostein, "Least-squares fitting of two 3D point sets", IEEE Trans. on Pattern Anal. and MI, vol. 5, pp. 698 - 700, 1987.

[55] Y. Y. Lee, A. Erdogan, and S.S. Rao, "How to assess regional and whole gut transit time with wireless motility capsule", Journal of Neurogastroenterology and Motility, vol. 20, no. 2, p. 265, 2014.

[56] A. M. Haase, T. Gregersen, V. Schlageter, M. S. Scott, M. Demierre, P. Kucera, and K. Krogh, "Pilot study trialing a new ambulatory method for the clinical assessment of regional gastrointestinal transit using multiple electromagnetic capsules", Journal of Neurogastroenterology \& Motility, vol. 26, no. 12, pp. 1783 - 1791, 2014. 